\title{
Influence of open trailing edge on laminar aerofoils at low Reynolds number
}

\author{
Rodolfo Sant', Luis Ayuso' and Jose Meseguer²
}

\begin{abstract}
This article deals with the effect of open trailing edge on the aerodynamic characteristics of laminar aerofoils at low Reynolds numbers, the attention being focussed on the influence of such a trailing-edge imperfection on the aerodynamic efficiency. Wind tunnel tests have been performed at different Reynolds numbers and angles of attack, and global aerodynamic as well as pressure distributions were measured (in these tests two types of open trailing edges, either sharp or rounded were considered). From experimental results, a quantitative analysis of the influence of the trailingedge thickness on the degradation of aerofoil aerodynamic performances has been obtained, which allows the establishment of a criterion for an acceptance limit for this kind of imperfection.
\end{abstract}

\section{Keywords}

Trailing edge, low-Reynolds, laminar aerofoil

\section{Introduction}

In this article, the influence of trailing-edge imperfections on the aerodynamic performances of classical laminar aerofoils is considered. Trailing-edge imperfections can arise during the manufacturing process in non-aeronautical applications, where manufacturing procedures are not so strict as in aeronautical applications. A typical example can be found in turbine blades, which usually are manufactured in two parts, or shells (the upper surface and the lower surface), and after assembled. In some cases, this last step is not performed properly, and the trailing edge becomes thicker than expected.

Normally, this kind of imperfection is corrected by reducing the trailing-edge thickness through sandpaper treatment, which implies a large man effort with the subsequent increase of production costs. Obviously, a not well-conditioned trailing edge means some deterioration of the aerodynamic performances of the resulting aerofoil. However, in spite of the relative importance of this problem, this performance deterioration is not very well documented in the literature.

Aiming to get some additional insight on this phenomenon, a test campaign was carried out to measure the influence of thick trailing edges on the aerodynamic loads on aerofoils at low Reynolds numbers. Low Reynolds number aerofoils have focussed the attention of many researchers during decades because of their use in a wide range of applications, from unmanned aerial vehicles (UAVs) to low power wind turbine blades, ${ }^{15}$ and also because their potential use in aircraft designed to flight in thin atmospheres like the one existing in Mars.

Leaving aside the use of thick or truncated trailing edges in transonic aerofoils, these configurations have been also used in small turbine and compressor blades to increase the robustness of the trailing edges of these devices.

As one could expect, thick trailing edges can produce a sensible increase of the maximum lift, but there is also a noticeable increase of the minimum aerodynamic drag. ${ }^{6}{ }^{9}$ The same criterion has been used to increase the mechanical strength of root aerofoils in turbine blades, with similar results with regards to lift and aerodynamic drag. ${ }^{10,11}$

\footnotetext{
'Departamento de Aerotecnia, Universidad Politécnica de Madrid, Spain ${ }^{2}$ Instituto Universitario 'Ignacio Da Riva', Universidad Politécnica de Madrid, Spain

Corresponding author:

Rodolfo Sant, Departamento de Aerotecnia, E.U.I.T. Aeronáutica, Universidad Politécnica de Madrid, Plaza del Cardenal Cisneros, 3, 28040 Madrid, Spain.

Email: rodolfo.sant@upm.es
} 
Some previous studies published elsewhere concerning non-laminar aerofoils at low Reynolds numbers show similar trends: ${ }^{12,13}$ the lift coefficient increases, but an important rise of the drag coefficient takes place, thus leading to a significant reduction of the aerodynamic efficiency.

To get a quantitative measure of the effect on aerodynamic performances of a gap between the upper and the lower surfaces of an aerofoil at the trailing edge, in the following paragraphs experimental results measured with a basic laminar aerofoil NACA $63_{2}-215$ are reported. The aerofoil model can be modified by increasing the dimensionless distance $\tau_{e}=t_{e} / c$ between the trailing edges of the lower and upper part of the aerofoil (where $t_{e}$ stands for the distance between both edges and $c$ for the nominal aerofoil chord). Experimental results show that beyond $\tau_{e}=0.05$ the degradation of aerofoil performances becomes unacceptable.

\section{Experimental set-up}

Experiments were performed at the Departamento de Aerotecnia of the Universidad Politécnica de Madrid by using a small open-circuit low-speed wind tunnel. The wind tunnel test chamber is $1.2 \mathrm{~m}$ high, $0.16 \mathrm{~m}$ wide and $1.5 \mathrm{~m}$ long. Test chamber is equipped with two windows (at the lateral walls of the test chamber), one of them being optically transparent. Upstream of the test section there is a contraction, with several screens at the contraction entrance, which provides a uniform low-turbulent incoming flow at the test chamber entrance. Flow uniformity in the test chamber, outside boundary layers, defined as the ratio to the mean velocity, $U_{\text {mean }}$, of the difference between the maximum, $U_{\max }$, and minimum, $U_{\min }$, velocities, that is $\left(U_{\max }-U_{\min }\right) / U_{\text {mean }}$, is less than $1 \%$, and the mean turbulence level is less than $0.5 \%$. Air velocity in the test section ranges from 5 to $30 \mathrm{~m} / \mathrm{s}$. Thus, Reynolds numbers up to $4.5 \times 10^{5}$, based on nominal aerofoil chord, $0.24 \mathrm{~m}$, can be reached. Maximum Mach number is 0.09 , so the flow behaves as incompressible and the air density is constant.

Two models of a laminar aerofoil NACA $63_{2}-215^{14}$ have been used for measurements, one of them for global aerodynamic load measurements (by using a three-component electronic balance), whereas the other model, which is equipped with 34 pressure taps distributed on the upper surface aerofoil, at its middle section (Figure 1), is devoted to pressure distribution measurements. Both models have been manufactured in a numerically controlled milling machine using an isotropic material widely used in modelling (Necuron ${ }^{\mathbb{B}}$ ), which allows a good surface finish for the models. Both aerofoil samples are provided with a hinge at the leading edge which allows the opening of the trailing edge.

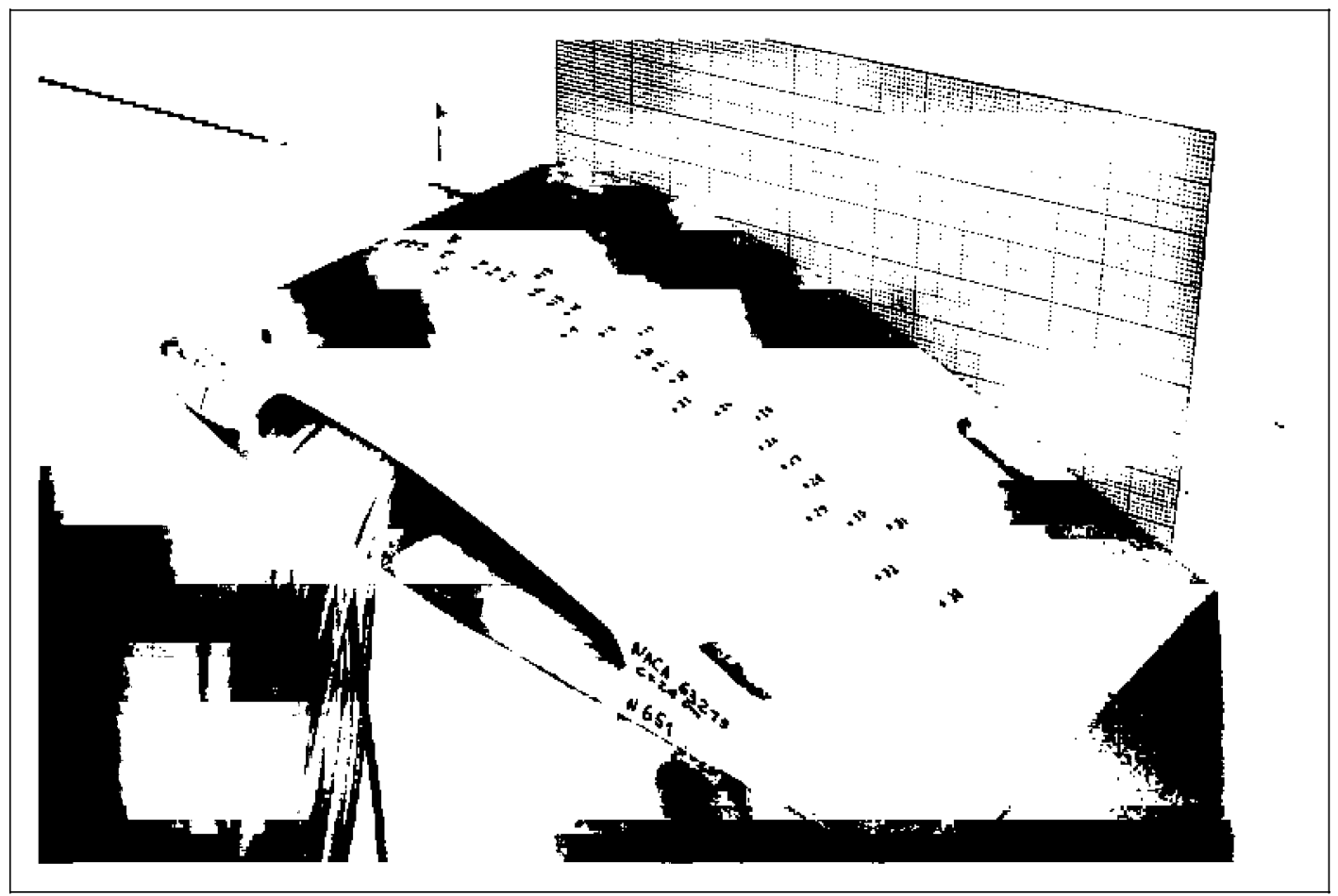

Figure I. View of the NACA $63_{2}-2 \mid 5$ model, with pressure taps. 
Model span is $15.8 \mathrm{~cm}$, whereas that of the wind tunnel test chamber wide is $16 \mathrm{~cm}$. No special provisions were undertaken to avoid air flow through the $1 \mathrm{~mm}$ gap between model and wind tunnel walls, nor to correct measured results to account for this gap effect, which is assumed to be small enough. Note that the gap width is much more smaller than the boundary layer thickness of wind tunnel sidewalls (in the section were the model is located the thickness of the boundary layer is from 10 to $15 \mathrm{~mm}$ depending on the Reynolds number). Besides the wall boundary layer effects, the Reynolds number based both on the potential flow velocity and on the gap between aerofoil and sidewalls ranges from 310 to 1900 , small enough to provide a high pressure drop to the flow in the gap, thus minimizing pressure gap effects.

The model is mounted at the center of the wind tunnel test section, which is $1.2 \mathrm{~m}$ high. Since the aerofoil chord is less than 0.25 times the height of the test chamber, and the angle of attack $\alpha$ has been kept within the range $|\alpha|<24^{\circ}$, no blockage corrections were performed. ${ }^{15}$ It must be emphasized that the aim of this work is not to measure the aerodynamic performances of open trailing-edge aerofoils, but to compare the effect of different aerofoil trailing-edge thicknesses on aerodynamic performances.

The forces were measured with a three-component electronic balance from PLINT Company. The balance is located on one of the side walls of the test chamber. Measured loads were lift, drag and pitching moment. The electronic balance load range was $100 \mathrm{~N}$ in lift forces, $50 \mathrm{~N}$ in drag forces and $3.1 \mathrm{Nm}$ in torque, and its accuracy was $0.015 \mathrm{~N}$ in lift forces, $0.0076 \mathrm{~N}$ in drag forces and $4.8 \times 10^{-4} \mathrm{Nm}$ in torque. At each angle of attack loads were measured at $10^{3} \mathrm{~Hz}$ sampling rate during $8 \mathrm{~s}$. Repeatability is good enough and uncertainty is low enough, obtaining values of RMS (root mean square) of 0.004 for lift coefficient, 0.002 for drag coefficient, and 0.001 for pitch moment coefficient. The model is connected to the electronic balance through a metallic bar which is located at point $1 / 4$ of upper surface part chord line, while the model of the lower surface is attached to the upper surface using four screws.

Pressure taps were connected to a pressure acquisition system DSA3217, from Scanivalve Corporation. Pressures were sampled at $500 \mathrm{~Hz}$ during $5 \mathrm{~s}$.

Models were tested in the range of angles of attack spanning from $-2^{\circ}$ to $24^{\circ}$, approximately. Four values of the Reynolds number have been considered: $0.75 \times 10^{5}, 1.5 \times 10^{5}, 3.0 \times 10^{5}$ and $4.5 \times 10^{5}$.

As already said, upper and lower aerofoil surfaces are hinged at the leading edge, so that any desired trailing-edge separation can be reached by using the appropriate gage, by rotating the lower surface aerofoil with respect to the upper one (Figure 2). Note that when a gap is established at the trailing edge, the resulting aerofoil is not a NACA $63_{2}-215$ exactly, but a thicker one with different locations of the maximum thickness, depending on the gage used. Two trailing-edge finishing were considered: sharp and rounded. For rounded trailing-edge tests, aerofoils are prolonged with a semicylindrical piece added all along the trailing edge of the model (Figure 2). The tested dimensionless trailing-edge gaps, $\tau_{e}=t_{e} / c$, were of $\tau_{e}=0.02,0.04,0.06$, 0.08 , and 0.10 .

The measured loads have been made dimensionless by using the aerofoil chord, $c$, and the upstream flow dynamic pressure, $q_{\infty}=\rho U_{\infty}^{2} / 2$, as characteristic magnitudes, where $\rho$ is the air density (around $1.12 \mathrm{~kg} / \mathrm{m}^{3}$ ) and $U_{\infty}$ stands for the incoming air speed. From load measurements, the variation with the aerodynamic angle of attack of the lift coefficient, $C_{l}=l /\left(q_{\infty} c\right)$, the drag coefficient, $C_{d}=d /\left(q_{\infty} c\right)$, the pitch moment coefficient, $C_{m}=m /\left(q_{\infty} c^{2}\right)$, as well as the lift-to-drag ratio, $C_{l} / C_{d}$, have been obtained. Here $l, d$ and $m$ are the lift, drag and pitching moment per unit span length, respectively.

Since in open trailing-edge aerofoil the aerofoil chord is not a properly defined topic, instead of the geometrical angle of attack, the aerodynamic angle of attack is used. Aerodynamic angle of attack is defined

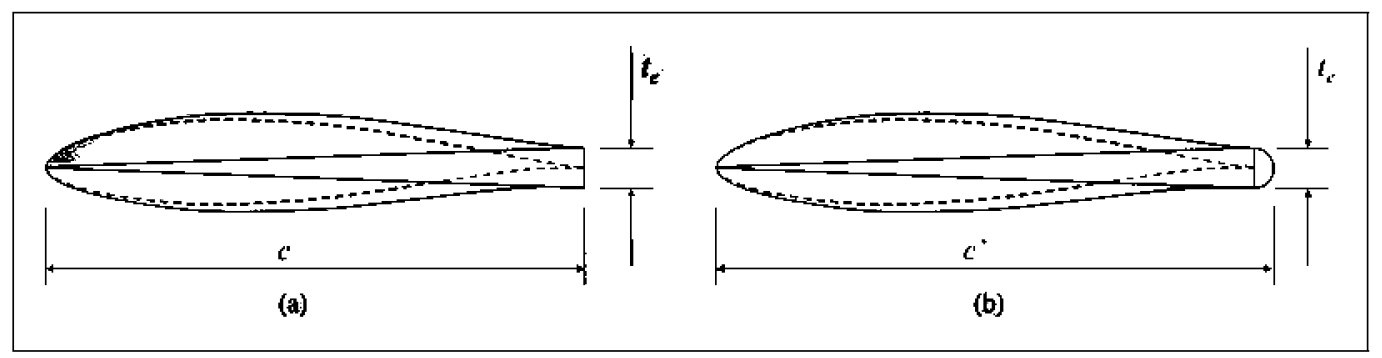

Figure 2. Sketches of the aerofoil model with trailing-edge modifications: upper and lower surfaces can be rotated around a leading edge hinge to obtain the desired trailing-edge gap, $\tau_{\mathrm{e}}$. (a) Sharp trailing edge, and (b) rounded trailing edge. Dotted lines represent nominal NACA $63_{2}-215$ aerofoil. 
as the angle between airstream direction and aerofoil zero lift direction.

From the measured upper surface pressure distributions, the pressure coefficient distributions were obtained. Pressure coefficient is defined as usual, $C_{p}=\left(p-p_{\infty}\right) / q_{\infty}$, where $p$ is the mean pressure measured on the tap under consideration, and $p_{\infty}$ and $q_{\infty}$ are the static and the dynamic upstream pressures, respectively.

\section{Results and discussion}

\section{Nominal aerofoil NACA $63_{2}-215$}

The results concerning the aerodynamic performances of the nominal aerofoil NACA $63_{2}-215$, lift coefficient, drag coefficient, lift-to-drag ratio and pitch moment at $1 / 4$ chord point versus aerodynamic angle of attack, are shown in Figure 3. In these plots the results corresponding to the above stated Reynolds number values $\left(0.75 \times 10^{5}, 1.5 \times 10^{5}, 3.0 \times 10^{5}\right.$, and $\left.4.5 \times 10^{5}\right)$ have been represented as well as the results corresponding to $R e=3.0 \times 10^{6}$ (obtained from Abbott and Von Doenhoff ${ }^{14}$ ). Although measurements have been performed both increasing and decreasing the angle of attack, since time gap between two consecutive measurements is large enough, no hysteresis has been detected, as it can be expected. According to experimental results, within the range of values of Reynolds number of the experiments here performed, the nominal aerofoil, NACA $63_{2}-215$, slightly increases the lift

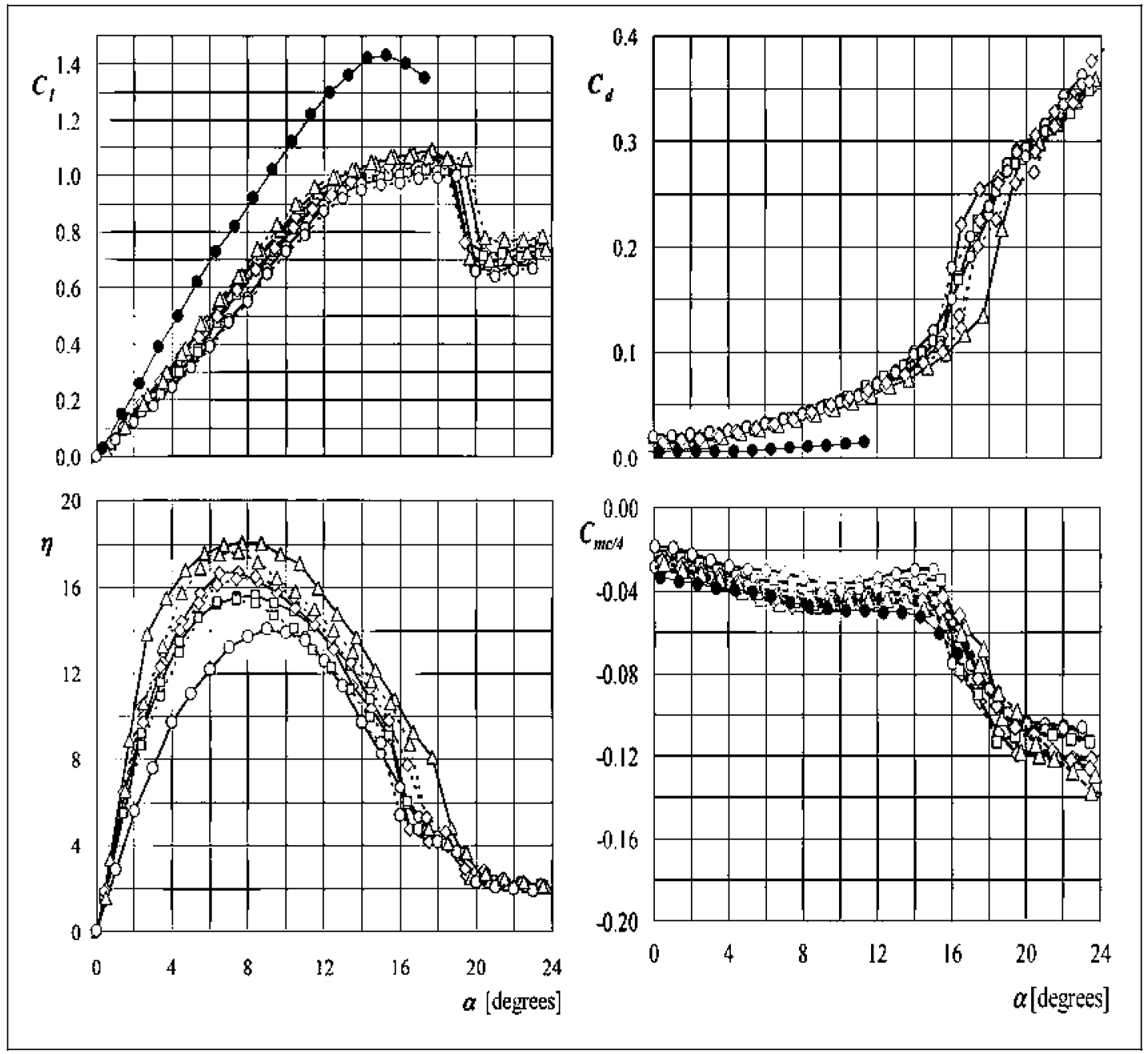

Figure 3. Aerodynamic performances of the nominal aerofoil NACA $63_{2}-215$. Variation with the aerodynamic angle of attack, $\alpha$, of the lift coefficient, $C_{1}$, drag coefficient, $C_{d}$, lift-to-drag ratio, $\eta=C_{1} / C_{d}$, and pitch moment at $1 / 4$ chord point, $C_{m c / 4}$. Symbols identify the value of the Reynolds number according to the following key: $R e=0.75 \times 10^{5}$ (circles); $1.5 \times 10^{5}$ (squares); $3.0 \times 10^{5}$ (rhombi); $4.5 \times 10^{5}$ (triangles); and $3.0 \times 10^{6}$ (black circles). Solid lines represent tests performed by increasing the angle of attack, whereas dotted lines indicate that the angle of attack decreases during the tests. 
coefficient, as well as the maximum lift coefficient, as Reynolds number grows. The lift curve slope also grows as Reynolds number grows. In all cases the linear behavior of the lift curve changes provided the aerodynamic angle of attack becomes high enough, and the lift curve slope becomes smaller when de angle of attack is larger than $12^{\circ}$, approximately. After a threshold value of the angle of attack (around $19^{\circ}$ ) the aerofoil stalls suddenly no matter the value of the Reynolds number is.

The drag coefficient of the nominal NACA $63_{2}-215$ aerofoil decreases as the Reynolds number increases, so that the aerodynamic efficiency (lift-to-drag ratio) increases, while the angle of attack of maximum efficiency decreases. On the other hand the $1 / 4$ chord pitching moment becomes more and more negative as the Reynolds number increases. The maximum aerodynamic efficiency of this cambered aerofoil is better than the one corresponding to a symmetric aerofoil for all values of the Reynolds number under consideration, at it can be deduced from the comparison of the result here presented with those concerning a NACA 0012 aerofoil reported elsewhere. ${ }^{12,13}$

It is worthwhile to compare load coefficiency measured at low Reynolds number with those resulting at high Reynolds number. In the same plot of Figure 3 the results corresponding to $R e=3.0 \times 10^{6}$ have been depicted. ${ }^{14}$ Obviously the aerodynamic performances of this aerofoil are significantly improved provided the Reynolds number is high enough, except in the case of the stall angle of attack, which becomes larger at low values of the Reynolds number.

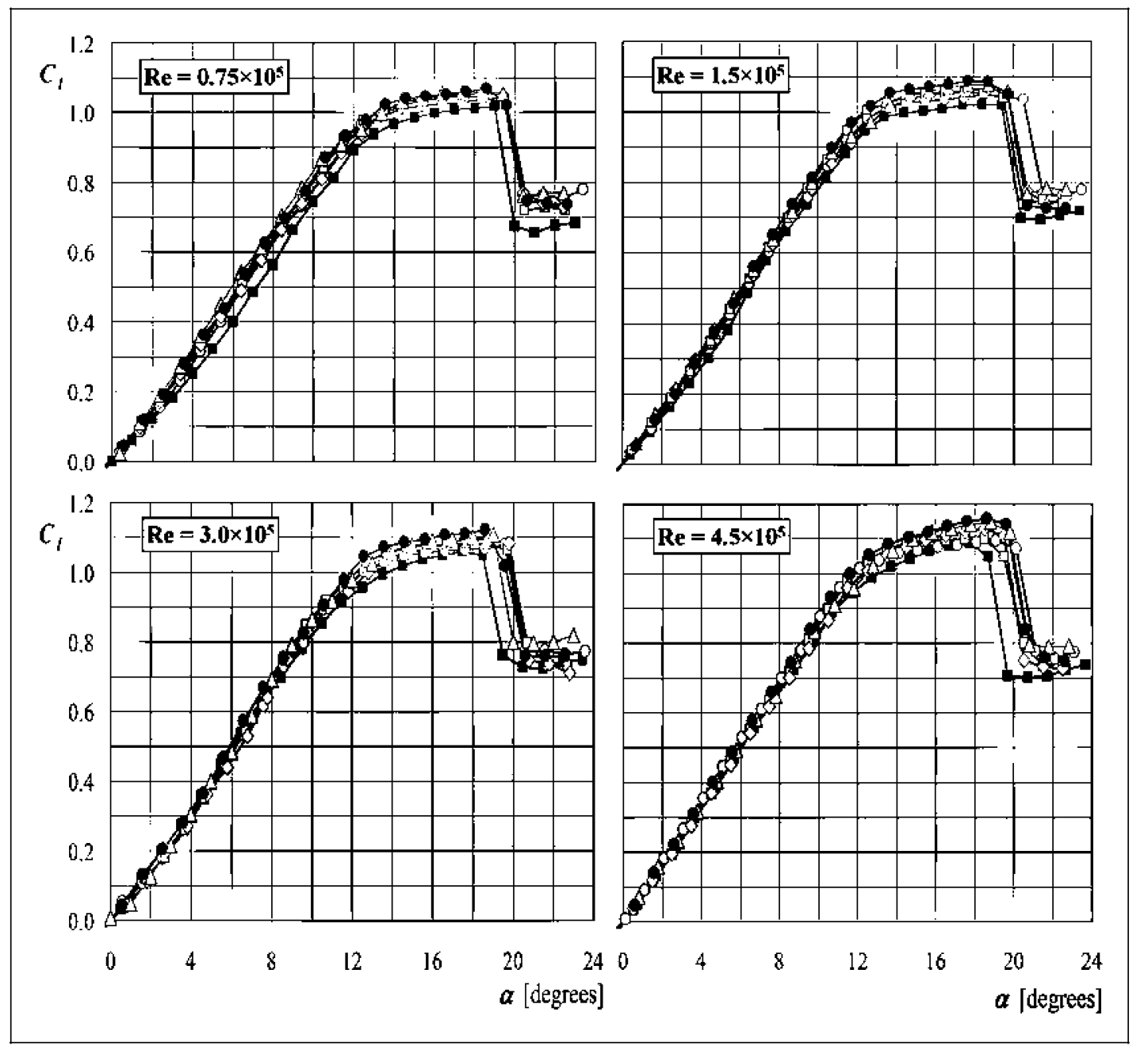

Figure 4. Variation with the aerodynamic angle of attack, $\alpha$, of the lift coefficient, $C_{l}$, of modified NACA $63_{2}-215$ aerofoils (sharp trailing edge). Symbols identify the value of the dimensionless trailing-edge gap according to the following key: nominal aerofoil (black squares); $\tau_{\mathrm{e}}=0.02$ (circles); 0.04 (squares); 0.06 (rhombi); 0.08 (triangles); and 0.10 (black circles). The values of the Reynolds number are indicated in the inserts. 


\section{Open trailing-edge aerofoils}

Concerning the influence of both the Reynolds number, $R e$, and the trailing-edge gap, $\tau_{e}$, the results obtained are summarized in Figure 4, where the variation of the aerofoil lift coefficient $C_{l}$ with the aerodynamic angle of attack $\alpha$ of sharp trailing-edge aerofoils is presented for different values of $R e$ and $\tau_{e}$. Independent of the value of the Reynolds number, the separation of the lower and upper surfaces at the trailing edge means an slightly increase of the maximum lift coefficient, the higher the value of the parameter $\tau_{e}$ the higher the growth of the lift coefficient, at least within the range of values of $\tau_{e}$ under consideration. Note that increments of the maximum lift coefficient close to $6 \%$ are obtained for $\tau_{e}=0.1$, independently of the value of the
Reynolds number. Note also that the lift coefficient grows linearly or almost linearly with the angle of attack until $\alpha \cong 12^{\circ}$, where a change of the lift slope curve appears independently of the values of the trailing-edge gap, $\tau_{e}$. If the angle of attack is increased further, the whole upper surface stalls and a sudden decrease of the lift coefficient takes place. The aerodynamic angle of attack to which the upper aerofoil surface becomes completely stalled increased slightly as the trailing-edge gap does.

Both above described phenomena can be explained by considering that, to some extent, a trailing-edge gap behaves like a small lower surface flap, which produces a small increment of lift when deflected, together with a noticeable increment of the aerofoil aerodynamic drag (as it can be observed in Figure 5, where similar plots to

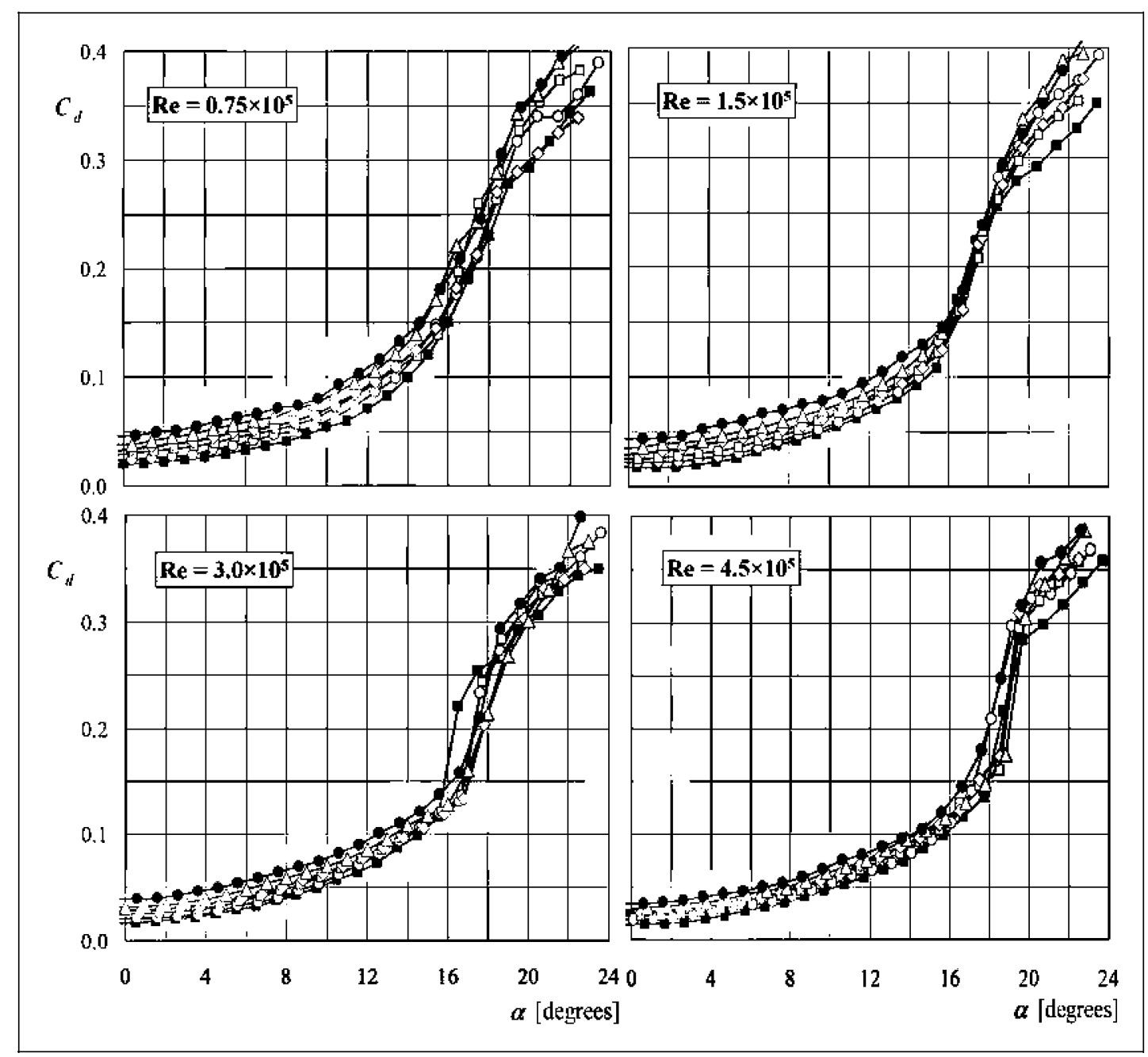

Figure 5. Variation with the aerodynamic angle of attack, $\alpha$, of the drag coefficient, $C_{d}$, of modified NACA $63_{2}-215$ aerofoils (sharp trailing edge). Symbols identify the value of the dimensionless trailing-edge gap according to the following key: nominal aerofoil (black squares); $\tau_{e}=0.02$ (circles); 0.04 (squares); 0.06 (rhombi); 0.08 (triangles); and 0.10 (black circles). The values of the Reynolds number are indicated in the inserts. 
those of Figure 4, but considering drag coefficients instead of lift coefficients, are presented).

Both force coefficients (lift and drag) can be merged in a single one: the aerodynamic efficiency or the lift-to-drag ratio, depicted in the plots included in Figure 6. As one could expect, the aerofoil efficiency decreases as the trailing-edge gap grows. The maxima of the different $\eta=C_{l} / C_{d}$ curves being reached at higher values of the angle of attack as $\tau_{e}$ increases, the magnitude of these maxima being smaller as $\tau_{e}$ grows.

To get some additional insight of the influence of the parameter $\tau_{e}$ in the degradation of the aerofoil performances, in Figure 7 the value $\eta_{\max }\left(\tau_{e}\right) / \eta_{\max }(0)-1$ has been represented. Here $\eta_{\max }(0)$ represents the maximum efficiency of the nominal aerofoil at the same Reynolds number. According to the data plot in Figure 7, this value varies almost linearly with the trailing-edge gap, $\eta_{\max }\left(\tau_{e}\right) / \eta_{\max }(0)-1 \cong-3.1 \tau_{e}$.

Assuming a gap between upper and lower surfaces exists at the trailing edge, a possibility to mitigate the degradation of aerofoil performances could be the

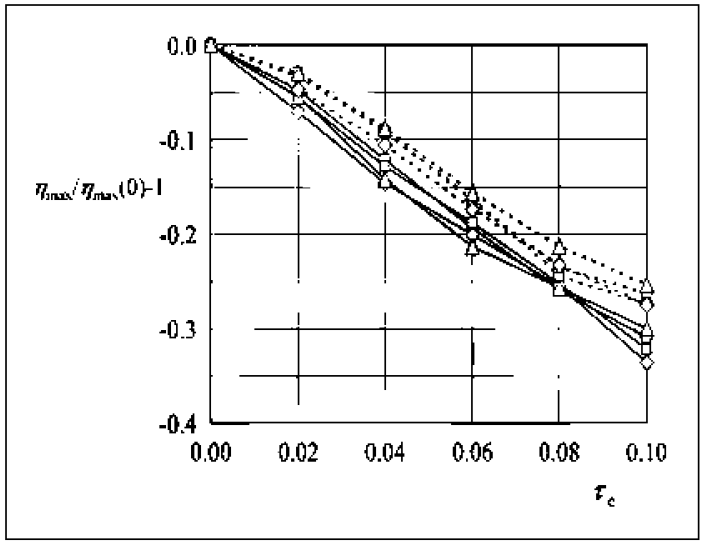

Figure 7. Variation with the dimensionless trailing-edge gap, $\tau_{\mathrm{e}}$, of the value $\eta_{\max }\left(\tau_{e}\right) / \eta_{\max }(0)-1$, where $\eta_{\max }\left(\tau_{e}\right)$ is the maximum aerodynamic efficiency of modified NACA $63_{2}-215$ aerofoils, and $\eta_{\max }(0)$ is the maximum aerodynamic efficiency of the nominal NACA $63_{2}-215$ aerofoil. Symbols identify the value of the Reynolds number according to the following key: $R e=0.75 \times 10^{5}$ (circles); $1.5 \times 10^{5}$ (squares); $3.0 \times 10^{5}$ (rhombi); and $4.5 \times 10^{5}$ (triangles). Type line indicates the type of trailing edge, sharp (solid lines) and rounded (dotted lines).

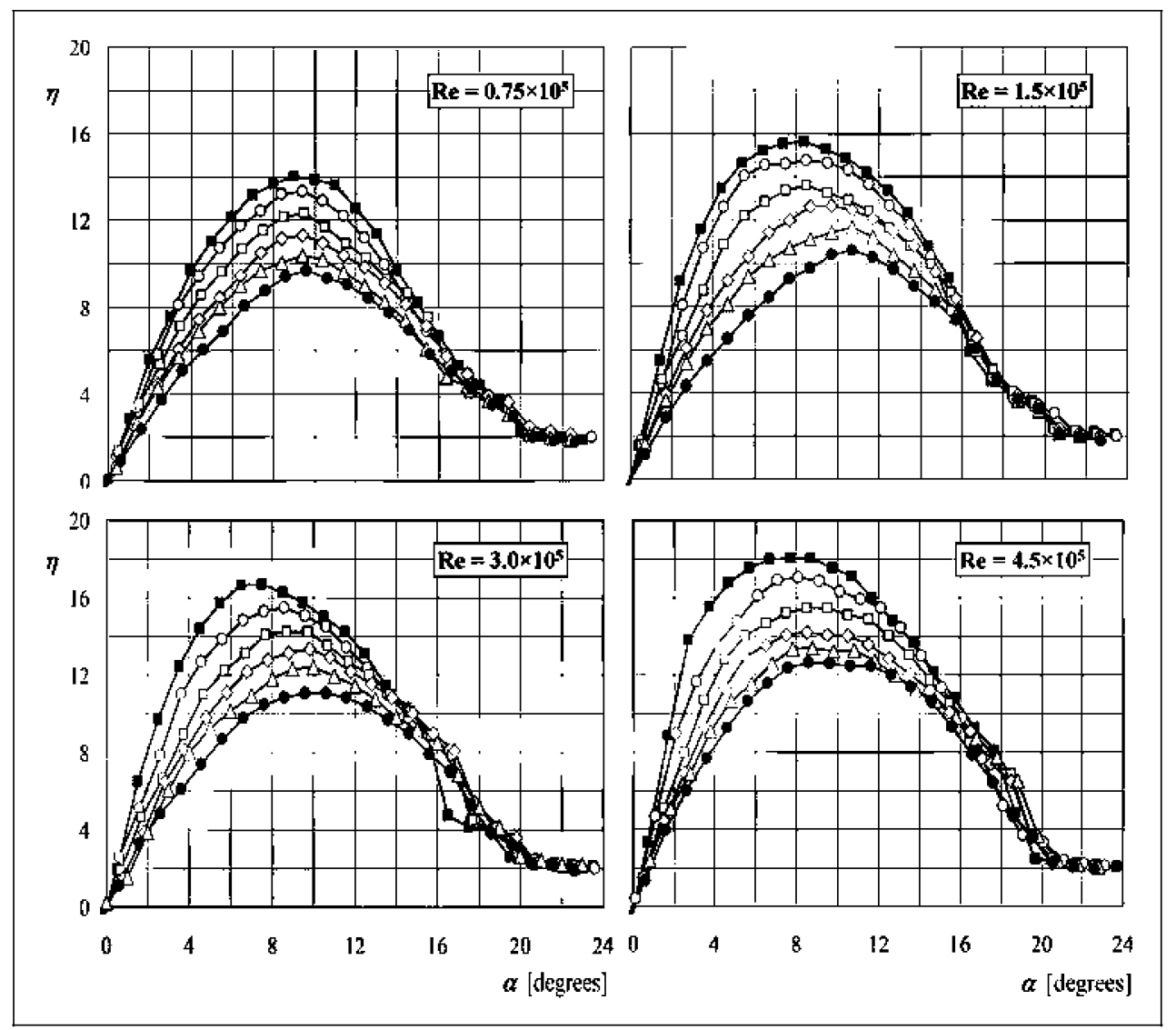

Figure 6. Variation with the aerodynamic angle of attack, $\alpha$, of the lift-to-drag ratio, $\eta=C_{l} / C_{d}$, of modified NACA $63_{2}-2 \mid 5$ aerofoils (sharp trailing edge). Symbols identify the value of the dimensionless trailing-edge gap according to the following key: nominal aerofoil (black squares); $\tau_{\mathrm{e}}=0.02$ (circles); 0.04 (squares); 0.06 (rhombi); 0.08 (triangles); and 0.10 (black circles). The values of the Reynolds number are indicated in the inserts. 
modification of the trailing edge by rounding it. This possibility has been experimentally analyzed by adding to the open trailing edge a half cylindrical bar whose cross section is a circumference $\tau_{e}$ in diameter. Note that with this approach the aerofoil chord becomes slightly larger, $c^{\prime}=c\left(1+\tau_{e} / 2\right)$ instead of $c$ (in this case, to made dimensionless the measured results the chord of the modified aerofoil, $c^{\prime}$, has been used as characteristic length). Five new aerofoils with this new configuration were tested, with $\tau_{e}$ ranging from 0.02 to 0.10 at 0.02 steps. The measured results, aerodynamic coefficients versus angle of attack for different values of $R e$ and $\tau_{e}$ are presented in Figure 8 (lift coefficient, $C_{l}$ ), in Figure 9 (drag coefficient, $C_{d}$ ), and in Figure 10 (liftto-drag ratio, $C_{l} / C_{d}$ ).

With regard to lift coefficients, the behavior is similar to that obtained in the sharp trailing-edge case, although now the lift increase due to a rounded trailing-edge gap is less pronounced that the one obtained with sharp edges. Even the increment of the drag coefficient is smaller, at least for small values of the dimensionless gap $\tau_{e}$, the reason being that, as it is known, a rounded trailing edge produces a narrower wake than a sharp one.

Because of the lift increment and the drag decrement, rounded trailing-edge aerofoils efficiencies are a little higher when compared with those of sharp trailing-edge aerofoils, as shown in Figure 7, in such a way that now the linear dependence on the gap parameter $\tau_{e}$ of the aerofoil efficiency can be written as $\eta_{\max }\left(\tau_{e}\right) / \eta_{\max }(0)-1 \cong-2.6 \tau_{e}$, some $16 \%$ smaller than the result obtained with sharp trailing edges. Hence, to round trailing edges when a gap between upper and lower aerofoil surfaces appears at the trailing

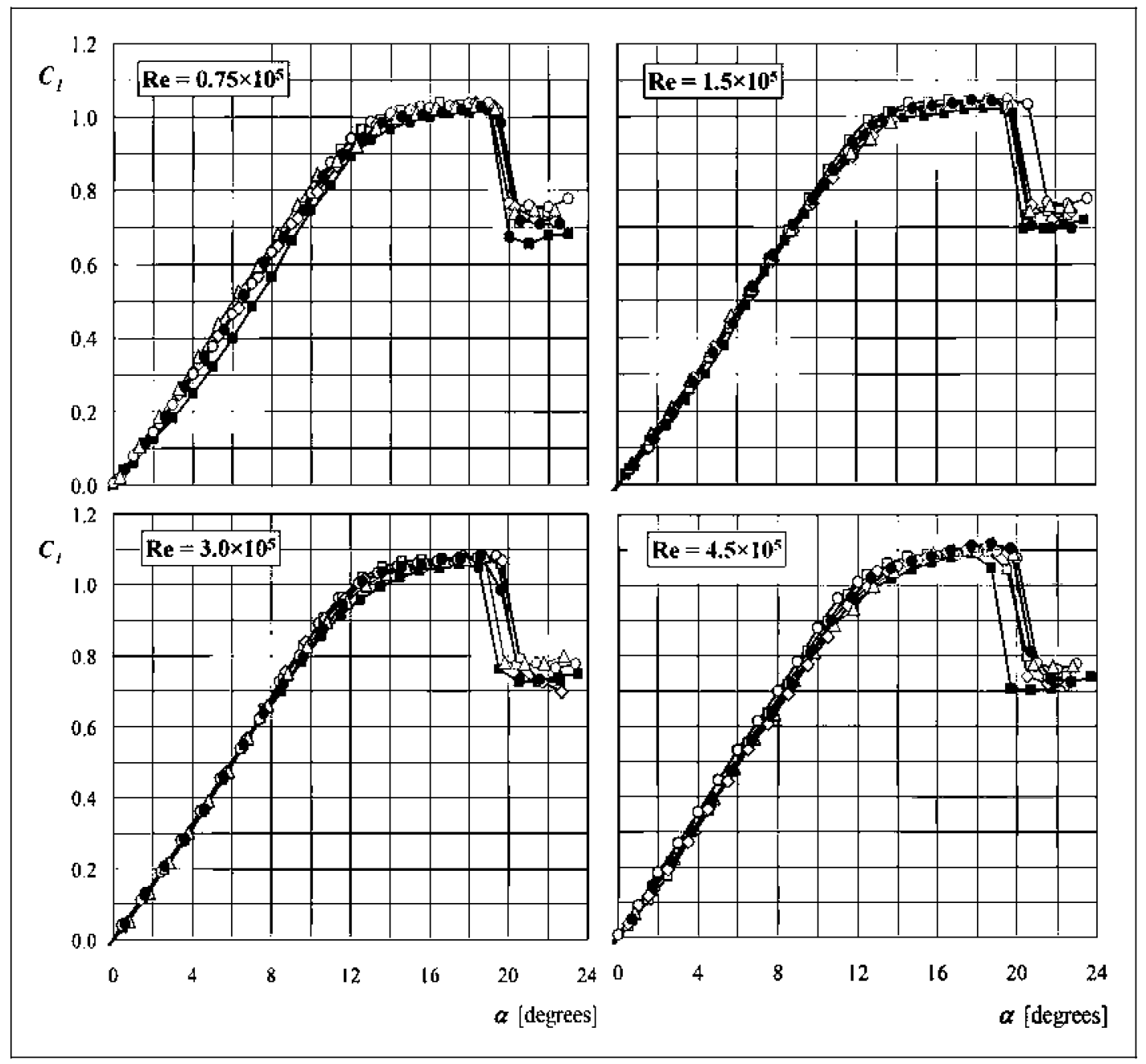

Figure 8. Variation with the aerodynamic angle of attack, $\alpha$, of the lift coefficient, $C_{l}$, of modified NACA $63_{2}-215$ aerofoils (rounded trailing edge). Symbols identify the value of the dimensionless trailing-edge gap according to the following key: nominal aerofoil (black squares); $\tau_{e}=0.02$ (circles); 0.04 (squares); 0.06 (rhombi); 0.08 (triangles); and 0.10 (black circles). The values of the Reynolds number are indicated in the inserts. 


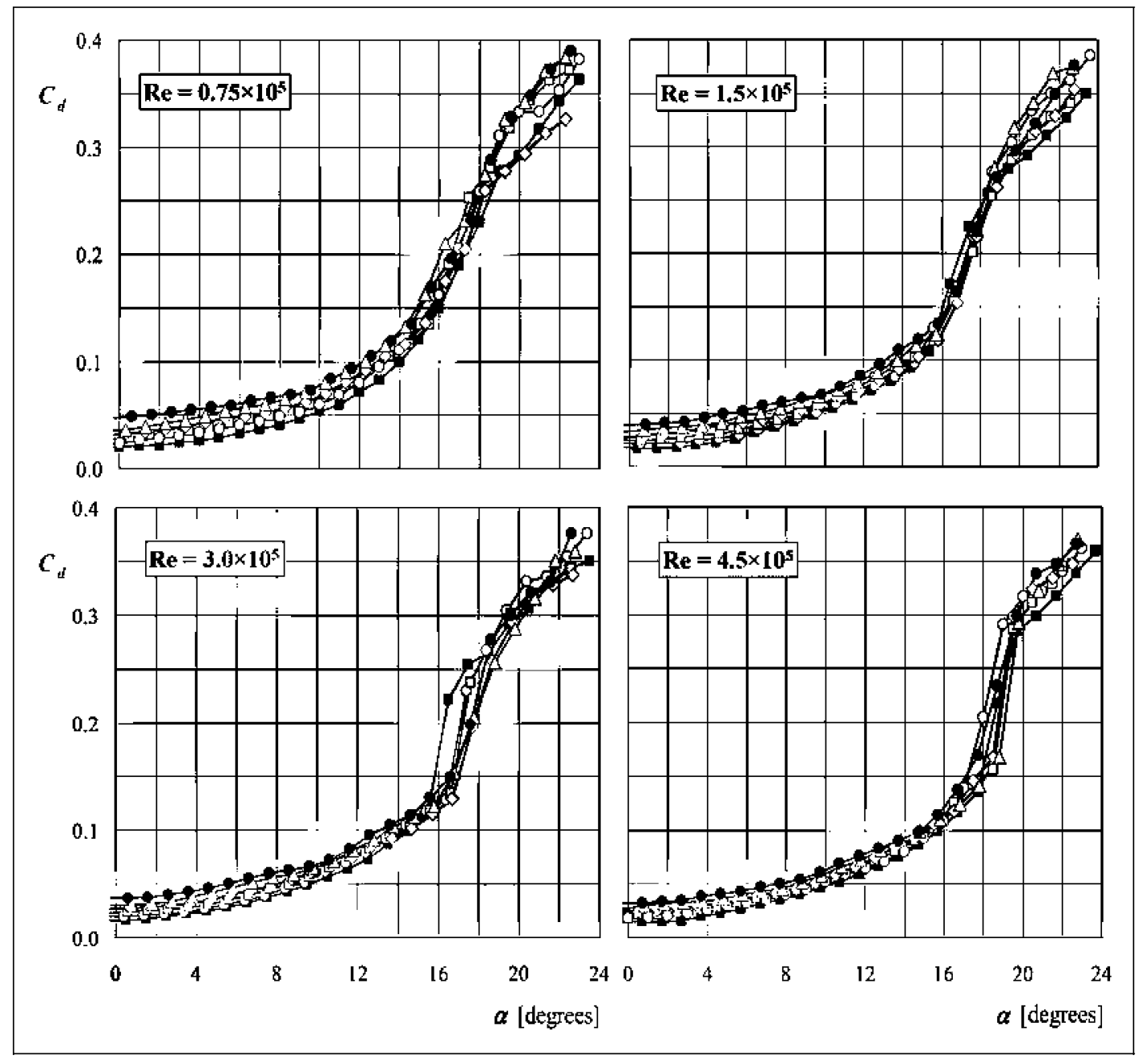

Figure 9. Variation with the aerodynamic angle of attack, $\alpha$, of the drag coefficient, $C_{d}$, of modified NACA $63_{2}-215$ aerofoils (rounded trailing edge). Symbols identify the value of the dimensionless trailing-edge gap according to the following key: nominal aerofoil (black squares); $\tau_{c}=0.02$ (circles); 0.04 (squares); 0.06 (rhombi); 0.08 (triangles); and 0.10 (black circles). The values of the Reynolds number are indicated in the inserts.

edge would be a method to alleviate aerofoil performances degradation.

\section{Pressure distribution tests on flat trailing-edge aerofoils}

To clarify the peculiarities of the flow morphology around the aerofoil, pressure distributions were measured by using the pressure model already described in the 'Experimental set-up' section.

Taking into account both Reynolds number and aerofoil geometry (dimensionless trailing-edge gap, $\tau_{e}$ ), one could expect a flow behavior mainly driven by leading edge geometry instead of trailing edge. Provided Reynolds number is small enough, it can be expected the formation of a recirculation bubble close to the leading edge, whose length along the upper surface aerofoil depends on the aerofoil thickness as well as on the angle of attack. ${ }^{3,16}{ }^{18} \mathrm{As}$ it is well known, in the case of medium and small thickness aerofoil flying at low Reynolds numbers, laminar boundary layer separation takes place close to the leading edge. The resulting shear layer becomes turbulent, which can lead to the reattachment of the boundary layer, although now as a turbulent boundary layer, thus forming a recirculation bubble at the aerofoil upper side (Figure 11) where it can be seen a high suction pressure near the leading edge followed by a plateau area and a sudden pressure recovery. The length of the plateau area specifies the length of the recirculation bubble.

As the angle of attack increases the boundary layer reattachment point moves toward the trailing edge, and 


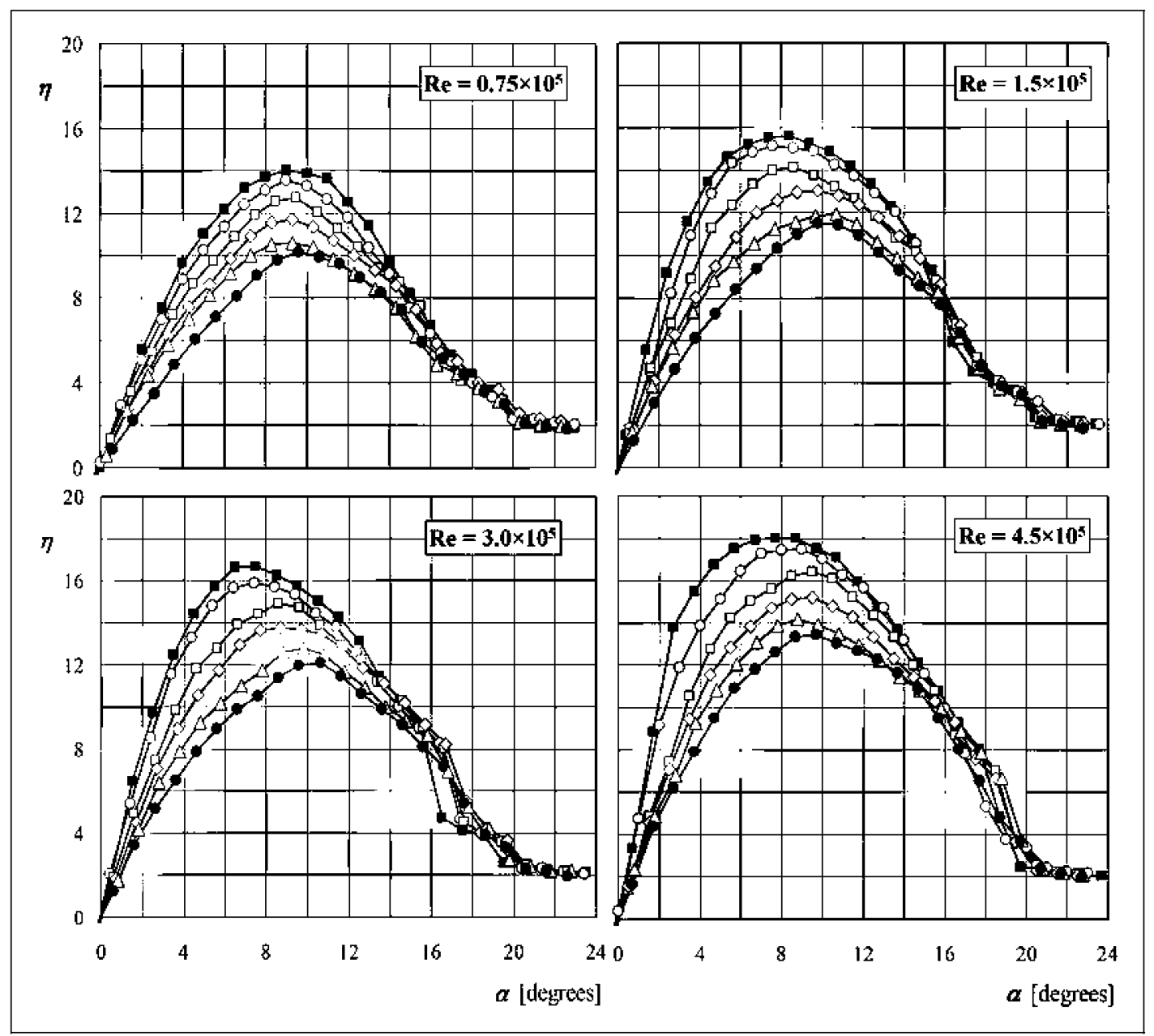

Figure 10. Variation with the aerodynamic angle of attack, $\alpha$, of the lift-to-drag ratio, $\eta=C_{d} / C_{d}$, of modified NACA $63_{2}-2 \mid 5$ aerofoils (rounded trailing edge). Symbols identify the value of the dimensionless trailing-edge gap according to the following key: nominal aerofoil (black squares); $\tau_{\mathrm{e}}=0.02$ (circles); 0.04 (squares); 0.06 (rhombi); 0.08 (triangles); and 0.10 (black circles). The values of the Reynolds number are indicated in the inserts.

the length of the recirculation bubble grows. For large values of the angle of attack the turbulent boundary layer separates at the trailing edge, indicated by the flat area at the end of pressure distributions. The turbulent boundary layer separation point moves toward the leading edge (shown by the increased length of the flat portion at the end) until it reaches the reattachment point of the recirculation bubble, and then the whole upper side aerofoil becomes stalled.

Since the formation of a recirculation bubble depends mainly on the adverse pressure gradients at the leading edge, the above described process is almost independent of the value of the of the dimensionless trailing-edge gap $\tau_{e}$, as can be observed in Figure 11, where the pressure distributions for several values of $\tau_{e}$ and Reynolds numbers are depicted. Note that, for a given Reynolds number, the variation of pressure distributions with the angle of attack is almost the same no matter the values of the trailingedge gap is.

\section{Conclusions}

Experiments performed with open trailing-edge aerofoils show that the most important effect on aerofoil performances is that the efficiency decreases as the gap between upper and lower surfaces at the trailingedge grows (the effect of such a gap on the lift-to-drag ratio can be mitigated by rounding the trailing edge).

On the other hand the lift coefficient slightly increases when the gap between upper and lower aerofoil surfaces at the trailing edge grows, this increment being noticeable for angles of attack beyond $10^{\circ}$ to $12^{\circ}$. Accordingly, the maximum lift coefficient also increases 


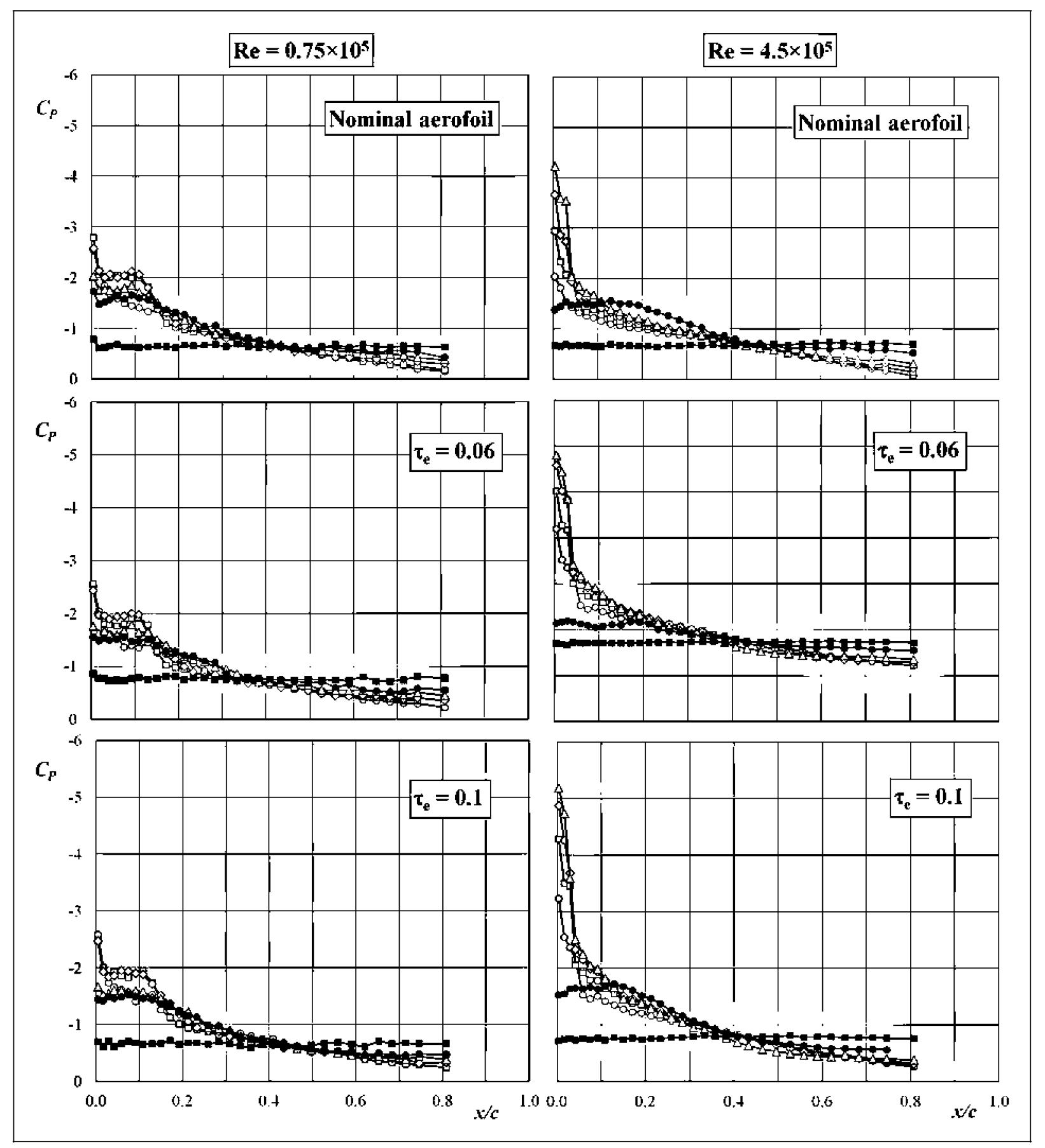

Figure II. Pressure coefficient along aerofoil upper side at angles of attack close to stalling at $\operatorname{Re}=0.75 \times 10^{5}$ (left column) and $4.5 \times 10^{5}$ (right column). Symbols identify the value of the angle of attack according to the following key: $10^{\circ}$ (circles); $12^{\circ}$ (squares); $14^{\circ}$ (rhombi); $16^{\circ}$ (triangles); $18^{\circ}$ (black circles); and $20^{\circ}$ (black squares). The values of the trailing-edge thickness are indicated in the inserts.

with the trailing-edge gap, independently of the value of the Reynolds number.

Associated to the trailing-edge gap is a remarkable increment of the aerodynamic drag, although the increment becomes smaller when the trailing edge is rounded instead of sharp. Therefore, since the increase of aerodynamic drag is larger than the one of lift, the aerofoil efficiency decreases.

The results show that small gaps at the trailing edge could be tolerated. Although this implies a reduction of the aerofoil efficiency, this could be compensated by the improvement of the maximum lift coefficient. As a 
guideline, considering unacceptable reductions over $15 \%$ in maximum aerodynamic efficiency, would be admissible trailing-edge gap up to $\tau_{e}=0.05$ for rounded trailing edge and up to $\tau_{e}=0.04$ in the case of sharp trailing edge.

\section{Funding}

This research received no specific grant from any funding agency in the public, commercial, or not-for-profit sectors.

\section{References}

1. Carmichael BH. Low Reynolds number airfoil survey. NASA CR-165803, Vol. 1, USA, 1981.

2. Nagamatsu HT and Cuche DE. Low Reynolds number aerodynamics characteristics of low-drag NACA 63-208 airfoil. $J$ Aircraft 1981; 18: 833-837.

3. Mueller TJ and Batill SM. Experimental studies of separation on a two-dimensional airfoil at low Reynolds number. $A I A A J 1982$; 20: 457-463.

4. Lissaman PBS. Low-Reynolds-number airfoils. Ann Rev Fluid Mech 1983; 15: 223-239.

5. Cebeci T. Essential ingredients of a method for low Reynolds number airfoils. AIAA $J$ 1989; 27: 1680-1688.

6. Ramjee V, Tulapurkara EG and Balabaskaran V. Experimental and theoretical study of wings with blunt trailing edge. $J$ Aircraft 1986; 23: 349-352.

7. Sato J and Sunada Y. Experimental research on blunt trailing-edge airfoil sections at low Reynolds number. AIAA J 1995; 33: 2001-2005.

8. Standish KJ and Van Dam CP. Analysis of blunt trailing edge airfoils. In: 41 st aerospace sciences meeting and exhibit, Reno, Nevada, USA, 2003.

9. Standish KJ and Van Dam CP. Aerodynamic analysis of blunt trailing edge airfoils. J Solar Energy Eng 2003; 125: 479-488.

10. Baker JP, Van Dam CP and Gilbert BL. Flatback airfoil wind tunnel experiment. Report, Sandia National Laboratories, Albuquerque, NM and Livermore, CA, USA, April 2008. Sandia Report SAND2008-2008.

11. Berg DE and Barone M. Aerodynamic and aeroacoustic properties of a flatback airfoil. WINDPOWER 2008. Texas, USA: Houston, 2008.

12. Sant R, Ayuso L and Meseguer J. Aerodynamic analysis of open trailing edge airfoils at low Reynolds number. In: 49th AIAA aerospace sciences meeting, 2011, Orlando, FL, USA.

13. Sant R, Ayuso L and Meseguer J. Aerodynamic study of airfoil geometric imperfections at low Reynolds number. In: 13th international conference on wind engineering ICWE13, Amsterdam, The Netherlands, 2011.

14. Abbott IH and Von Doenhoff AE. Theory of wing sections. New York: Dover Publications Inc., 1959.

15. Allen HJ and Vincenti WG. Wall interference in a twodimensional-flow wind tunnel, with consideration of compressibility. NACA Report no. 782, USA, 1944.

16. Gault DE. An experimental investigation of regions of separated laminar flow. NACA TN-3505, USA, 1955.

17. Tani I. Low-speed flows involving bubble separation. Progr Aeronaut Sci 1964; 5: 70-103.

18. Brendel $M$ and Mueller TJ. Boundary-layer measurements on an airfoil at low Reynolds numbers. $J$ Aircraft 1988; 25: 612-617.

\section{Appendix}

\section{Notation}

$c$

$c^{\prime}$

$C_{d}$

$C_{l}$

$C_{m}$

$C_{m \mathrm{c} / 4}$

$d$

l

m

$p$

$p_{\infty}$

$q_{\infty}$

Re

$t_{e}$

$U_{\max }$

$U_{\text {mean }}$

$U_{\text {min }}$

$U_{\infty}$

\section{$\alpha$}

$\eta$

$\eta_{\max }$

$\rho$

$\tau_{e}$ nominal aerofoil chord length modified aerofoil chord length drag coefficient

lift coefficient

pitch moment coefficient

pitch moment at $1 / 4$ chord point

drag force

lift force

pitch moment

local pressure

static upstream pressure

dynamic pressure

Reynolds number

aerofoil trailing-edge thickness

test chamber maximum velocity

test chamber mean velocity

test chamber minimum velocity

test chamber air speed

aerodynamic angle of attack

aerodynamic efficiency

maximum aerodynamic efficiency

air density

dimensionless trailing-edge thickness 